\title{
Safety in medication use
}

\section{Mary P. Tully and Bryony Dean Franklin: safety in medication use, 2016, CRC Press-Taylor and Francis Group, Boca Raton, USA. ISBN: 978-1-4822-2700-0}

\author{
J. W. F. van Mil ${ }^{1}$
}

Received: 6 April 2016/Accepted: 13 May 2016/Published online: 19 May 2016

(C) Springer International Publishing 2016

Over the last decades, medication errors have become an important part of the discussions around medication use processes. This is not only due to a better analysis of the prescribing, dispensing and medicine use process as a result of the report 'To err is Human' (2000, Institute of Medicines, USA), but also to the realisation that drug-related problems may prevent optimal outcomes. Traditionally, the focus of most pharmacists is on the prescribing and dispensing process, and this tradition can also be recognised in this book. In fact, the term 'medication error' which is defined as preventable harm relating to medication use in clinical practice, is used especially in those settings. The terms drug-related problems and drug therapy problems, especially used when discussing medicine use, are less visible in this book.

Mary Tully and Bryony Dean Franklin coordinated the contribution of 50 people from over 10 countries, including three 'patients', to tell the story about safety in medication use, and focussed on technology and professional behaviour. In three main sections: Problems in the Medication Use Process, Approaches to understanding and resolving the problems, and Putting the solutions into practice, they elaborate the impact of medication errors and provide potential solutions.

The book delivers an overview about both theory and practice, from different perspectives. It discusses all kinds of novel technical solutions to solve medication safety problems, including electronic prescribing, the use of barcodes and automated dispensing. Especially in the second chapter, important theoretical background is given which helps to understand the third chapter, where solutions are discussed.

This book will especially be of value for health-system and hospital pharmacists, not so much for community pharmacists (who naturally have a role in medication safety). There also will be an important place for this book in the pharmacy education. From the perspective of the daily practice of clinical pharmacists, the book is not very helpful for their direct patient care. Medication reconciliation is discussed as a technical procedure, but not the clinical medication review. Pharmaceutical care nor medication management (or whatever term you want to use) seem to be a leading topic. Quality of prescribing, and quality of drug-use are just mentioned as a source for professional errors, not as concern for medicine users. In vain one will search in the book for important information on patient or medicine-user behaviour like adherence or patient ADR reporting, or on the patient-care giver interactions like in counselling and creating awareness.

The authors should nevertheless be complimented on making such as broad and important contribution to the profession of pharmacy.

\footnotetext{
$\triangle$ J. W. F. van Mil

jwfvmil@planet.nl

1 Zuidlaren, The Netherlands
} 